\title{
Changes in MiRNA-5196 Expression as a Potential Biomarker of Anti- TNF-a Therapy in Rheumatoid Arthritis and Ankylosing Spondylitis Patients
}

\author{
Marzena Ciechomska ${ }^{1,2} \cdot$ Krzysztof Bonek $^{3} \cdot$ Michal Merdas $^{2} \cdot$ Patryk Zarecki $^{2} \cdot$ Jerzy Swierkot $^{4} \cdot$ Piotr Gluszko $^{3}$. \\ Katarzyna Bogunia-Kubik ${ }^{2,5}$. Wlodzimierz Maslinski ${ }^{1}$
}

Received: 6 November 2017 / Accepted: 10 April 2018 / Published online: 9 May 2018

(c) The Author(s) 2018

\begin{abstract}
In this study, we analysed the expression level of sera circulating miRNA-5196 in rheumatoid arthritis (RA) and ankylosing spondylitis (AS) patients before and after tumor necrosis factor (TNF)- $\alpha$ therapy as biomarkers predicting positive treatment outcome. We enrolled 10 RA patients, 13 AS patients, and 12 healthy individuals in the study. The expression of miRNA5196 was measured by real-time polymerase chain reaction before and after anti-TNF- $\alpha$ therapy. Disease activity of RA patients was assessed using disease activity score 28 (DAS28), whereas ankylosing spondylitis DAS (ASDAS) was used in AS patients. MiRNA-5196 expression was significantly higher in patients with RA and AS before TNF- $\alpha$ therapy than in those following anti-TNF- $\alpha$ therapy and healthy controls. Changes in miRNA-5196 expression positively correlated with delta DAS28 or delta ASDAS, respectively, following TNF- $\alpha$ therapy. In contrast, changes in C-reactive protein (CRP) levels in RA and AS patients did not positively correlate with DAS28 or ASDAS changes. Receiver-operating characteristic analysis showed better diagnostic accuracy of miRNA-5196 expression both in RA (area under curve (AUC) $=0.87, p=0.055)$ and AS patients ( $\mathrm{AUC}=0.90, p=0.050$ ) compared to CRP levels in $\mathrm{RA}(\mathrm{AUC}=0.75, p=0.201$ ) and AS patients (AUC $=0.85$, $p=0.086$ ) upon biologic therapy treatment. Finding novel biomarkers, including miRNA-5196 which allow to predict and monitor anti-TNF- $\alpha$ response, would be of clinical value especially during the early phase of RA or AS development.
\end{abstract}

Keywords MiRNA $\cdot$ Biomarker $\cdot$ Rheumatoid arthritis $\cdot$ Ankylosing spondylitis $\cdot$ Anti-TNF- $\alpha \cdot$ Biologic therapy

\section{Introduction}

Marzena Ciechomska

m.m.ciechomska@gmail.com

1 Department of Pathophysiology and Immunology, National Institute of Geriatrics Rheumatology and Rehabilitation, Warsaw, Poland

2 Laboratory of Clinical Immunogenetics and Pharmacogenetics, Hirszfeld Institute of Immunology and Experimental Therapy, Polish Academy of Sciences, Wroclaw, Poland

3 Department of Rheumatology, National Institute of Geriatrics Rheumatology and Rehabilitation, Warsaw, Poland

4 Department of Rheumatology and Internal Medicine, Wroclaw Medical University, Wroclaw, Poland

5 Department of Internal, Occupational Diseases, Hypertension and Clinical Oncology, Wroclaw Medical University, Wroclaw, Poland
Rheumatic diseases, including rheumatoid arthritis (RA), systemic sclerosis (SSc), or ankylosing spondylitis (AS), are chronic autoimmune disorders characterized by pain and joint inflammation (Ciechomska and O'Reilly 2016). In the industrialized world, rheumatic diseases affect more individuals than any other disease group (eular.org https ://www.eular.org/myUploadData/files/10\%20things\%20 on\%20RD.pdf). In fact, a third of people of all ages are affected by rheumatic diseases at some point during their lifetime (eular.org https://www.eular.org/myUploadData/ files/10\%20things\%20on\%20RD.pdf). Rheumatic diseases tend to be progressive in terms of disability; therefore, during the last decade, the economic burden of rheumatic diseases has been increasingly recognised. The cost of rheumatic diseases is estimated at more than 200 billion euros per year in Europe (eular.org https://www.eular.org/myUpl oadData/files/10\%20things\%20on\%20RD.pdf). In particular, 
RA affects approximately $1 \%$ of Western countries and without optimal treatment approximately $30 \%$ of patients with RA become permanently work disabled within 2-3 years of diagnosis (Gibofsky 2012). Unfortunately, there is no cure for rheumatic diseases. Treatment with conventional disease modifying anti-rheumatic drugs (cDMARDs) and/ or nonsteroidal anti-inflammatory drugs (NSAIDs) is often associated with various adverse reactions, thus introduction in 1998 of biologic drug therapies provided a new form of treatment (Curtis and Singh 2011). Tumor necrosis factor (TNF)- $\alpha$ plays a pivotal role in tissue destruction and overall RA pathogenesis; therefore, the development of anti-TNF- $\alpha$ therapy has been a milestone in the treatment of RA. Based on experimental and clinical evidences, blockade of TNF- $\alpha$ with antibodies resulted in down-regulation of interleukin (IL)-1, granulocyte-macrophage colony-stimulating factor, IL-6, IL-8 and many other active molecules such as matrix metalloproteinases (MMPs) (Catrina et al. 2002; Maini and Feldmann 2002; Taylor and Feldmann 2009). These factors are involved in joint destruction in RA patients (Brennan et al. 1989). Similarly, patients suffering from AS have shown improvements in pain, functional ability and inflammatory markers such as $\mathrm{C}$ reactive protein (CRP) upon antiTNF- $\alpha$ treatment (Bao et al. 2014; Inman et al. 2008; Wang et al. 2016). In addition, global gene expression studies of AS patients demonstrated that genes involved in Toll-like receptor signaling, TNF signaling, type I interferon signaling and wingless-type MMTV integration site family (Wnt) signaling returned to normal levels following TNF- $\alpha$ inhibitors treatment (Dolcino et al. 2017). These data strongly suggest beneficial role of anti-TNF- $\alpha$ therapy both in RA and AS patients. Unfortunately, the high cost of treatment and the degree of efficacy of anti-TNF- $\alpha$ treatment pose significant problems and fail to provide a solution to all patients (Nair et al. 2016; Schoels et al. 2010). Therefore, there is a strong need to identify the factors which allow to predict a successful outcome before the start of therapy not only in patents suffering for RA but also other rheumatic diseases including AS.

MicroRNAs (miRNAs) are endogenous, non-coding, single-stranded RNAs of approximately 19-25 nucleotides in length. They can negatively regulate gene expression of target messenger RNA (mRNA) (Ciechomska et al. 2014; Macfarlane and Murphy 2010). In addition, miRNAs are attractive as potential biomarkers, since their abnormal expression pattern reflects the underlying pathophysiologic processes (Alevizos and Illei 2010). The expression of miRNAs can be altered under conditions of pathophysiological stress, disease or treatment (Mendell and Olson 2012). Thus, identification of miRNAs which will be effective as biomarkers determining early diagnosis and response to anti-TNF- $\alpha$ therapy might be of great interest in RA and AS. In this study, we evaluated whether changes in miRNA-5196 expression can be used as a biomarker predicting the positive outcome of anti-TNF- $\alpha$ therapy in RA and AS patients.

\section{Materials and Methods}

\section{Sample Collection and Cell Purification}

Ten patients who fulfilled the American College of Rheumatology (ACR) and the European League Against Rheumatism (EULAR) criteria (Aletaha et al. 2010) for the classification of RA were obtained from Wroclaw Medical University. Their clinical characteristics are summarized in Table 1. Thirteen patients who fulfilled the classification of ASAS criteria from 2009 for the diagnosis of AS (Rudwaleit et al. 2009) were obtained from National Institute of Geriatrics Rheumatology and Rehabilitation in Warsaw. Their clinical characteristics are summarized in Table 2. These studies were approved by the local ethics committees (approval no. 335/2014 and KBT-1/6/2017) and all RA and AS patients provided fully informed written consent. Fifteen healthy donors with no history of autoimmune disease were included as healthy control (HC). The blood from $\mathrm{HC}$ was collected from a local blood donor centre or directly from healthy volunteers. The serum samples from HC, RA and AS patients were collected in serum separation tubes (BD Vacutainer ${ }^{\circledR}$ SST II Plus), aliquoted and frozen at $-80^{\circ} \mathrm{C}$. To determine correct disease activity score 28 (DAS28) in RA patients, the level of CRP was used in formula. Similarly, CRP was used as one of the parameters to estimate the ASDAS score.

Table 1 Clinical and laboratory data of RA patients

\begin{tabular}{ll}
\hline Parameters of RA patients $(n=10)$ & RA patients \\
\hline Age, years, median (range) & $59(27-74)$ \\
Sex F/M & $6 / 4$ \\
Disease duration, years, median (range) & $7(2-25)$ \\
Anti-CCP Abs \% $(n)$ & $30 \%(n=3)$ \\
RF \% $(n)$ & $90 \%(n=9)$ \\
CRP, mg/L, median (range) before anti-TNF- $\alpha$ & $10.15(1.9-30.2)$ \\
CRP, mg/L, median (range) after anti-TNF- $\alpha$ & $3.4(3.4-27.9)$ \\
BMI median (range) & $24(18-25)$ \\
Smoker, \% ( $n)$ & $0 \%(n=0)$ \\
Treatment & \\
Etanercept, \% ( $n$ ) & $70 \%(n=7)$ \\
Adalimumab, \% ( $n$ ) & $30 \%(n=3)$ \\
Methotrexate, $\%$ (dose of treated patients) & $100 \%(10-25$ mg) \\
Average DAS28 before treatment (range) & $6.77(6.16-7.77)$ \\
Average DAS28 after treatment (range) & $4.48(3.69-5.31)$ \\
\hline
\end{tabular}


Table 2 Clinical and laboratory data of AS patients

\begin{tabular}{ll}
\hline Parameters of AS patients $(n=13)$ & AS patients \\
\hline Age, years, median (range) & $50(32-59)$ \\
Sex F/M & $10 / 3$ \\
Disease duration, years, median (range) & $9.5(1-37)$ \\
HLA-B27\% ( $n)$ & $77 \%(n=10)$ \\
Iritis $(n)$ & $31 \%(n=4)$ \\
CRP, mg/L, median (range) before anti-TNF- $\alpha$ & $7(3-61)$ \\
CRP, mg/L, median (range) after anti-TNF- $\alpha$ & $6(2-15)$ \\
BMI median (range) & $23(18-25)$ \\
Smoker, \% ( $n)$ & $23 \%(n=3)$ \\
Treatment & \\
Golimumab, \% $(n)$ & $15 \%(n=2)$ \\
Adalimumab, \% ( $n$ ) & $77 \%(n=10)$ \\
Certolizumab, $\%(n)$ & $8 \%(n=1)$ \\
Average ASDAS before treatment (range) & $3.64(1.8-4.8)$ \\
Average ASDAS after treatment (range) & $2.29(1-3.5)$ \\
Average BASDAI before treatment (range) & $6.10(2.8-8.8)$ \\
Average BASDAI after treatment (range)s & $3.48(1-8)$ \\
Average HAQ before treatment (range) & $1.19(0.37-3.75)$ \\
Average HAQ after treatment (range) & $0.57(0-2.12)$ \\
\hline
\end{tabular}

\section{Gene Expression Study}

The volume of $300 \mu \mathrm{l}$ of sera from HC, RA and AS patients was used to isolate circulating miRNA-5196 using NucleoSpin ${ }^{\circledR}$ miRNA plasma/serum (Macherey-Nagel, Germany) according to the manufacturer's protocol. TaqMan (®) microRNA RT Kit (Thermo Fisher Scientific, USA) was used to reverse transcribe to cDNA with the use of TaqMan ${ }^{\circledR}$ MicroRNA Assays for hsa-miR-5196-5q (471527_mat), and hsa-let-7a (000377) all from Thermo Fisher Scientific (USA). Based on previously published papers, hsa-let-7a was used as an internal control to normalize miRNA-5196 input (Davoren et al. 2008; Li et al. 2015). The $20 \mu \mathrm{L} \mathrm{PCR}$ reaction included $1.33 \mu \mathrm{L}$ RT product, $1 \times$ TaqMan Universal PCR master mix and $1 \mu \mathrm{L}$ primers and probe mix of the TaqMan MicroRNA Assay Kit (Thermo Fisher Scientific, USA). Reactions were performed at $95{ }^{\circ} \mathrm{C}$ for $10 \mathrm{~min}$, followed by 50 cycles at $95{ }^{\circ} \mathrm{C}$ for $15 \mathrm{~s}$ and $60{ }^{\circ} \mathrm{C}$ for $1 \mathrm{~min}$. Samples were analysed in triplicate using the Viia 7 or QuantStudio 5 qRT-PCR machines (Thermo Fisher Scientific, USA). The expression levels relative to the average $\mathrm{HC}$ (arbitrarily set at 1 ) were calculated using the following equation: $\left(2^{\wedge}\right.$ Delta Delta CT $)-1$, all normalized to hsa-let$7 \mathrm{a}$ internal control.

\section{Statistical Analysis}

All data are presented as mean \pm SEM. D'Agostino \& Pearson normality test was used to confirm the use of parametric or non-parametric test for the further analysis. The differences between the groups were tested for their statistical significance using either $t$ test or Mann-Whitney $U$ test. A $p$ value of less than 0.05 was considered statistically significant; $p$ values are expressed as follows: ns for not significant; $0.05>p>0.01$ as *; $0.01>p>0.001$ as $* * ; p<0.001$ as $* * *$. Correlations between delta DAS28 or ASDAS and delta miRNA-5196 and delta CRP following TNF- $\alpha$ therapy in RA or AS patients, respectively, were analysed using the non-parametric correlation Spearman analysis. To validate the accuracy of biomarkers, receiver-operating characteristic (ROC) curve analysis was introduced. GraphPad Prism (GraphPad Software) was used to calculate all tests. The cut-off value was established based on the Youden Index which allows to demonstrate the maximum potential effectiveness of a biomarker.

\section{Results}

\section{The Expression of Sera Circulating MiRNA-5196 is Elevated in Patients with Autoimmune Rheumatic Diseases Including SSc, RA and AS Compared to HC}

Since we have previously demonstrated that the level of miRNA-5196 is increased in sera and monocytes isolated from SSc patients compared to HC (Ciechomska et al. 2017), we sought to investigate the level of miRNA-5196 in sera from RA and AS patients to determine whether circulating miRNA-5196 can be used as a potential biomarker of rheumatic diseases. As a positive control of increased miRNA5196 expression we used SSc sera. Indeed, the expression of SSc miRNA-5196 was significantly enhanced (3.57-fold, $p=0.006)$ compared to HC sera. The level of circulating miRNA-5196 was also significantly elevated in other rheumatic diseases. In RA and AS patients, the level of miRNA5196 was 4.72 -fold $(p=0.0001)$ and 4.41 -fold $(p=0.0003)$, increased, respectively compared to $\mathrm{HC}$ sera (Fig. 1). Of note, in those RA patients who were undergoing 6 months anti-TNF- $\alpha$ therapy, the level of circulating miRNA-5196 was decreased but still significantly higher than HC (2.18fold, $p=0.012$,). In AS patients treated with TNF- $\alpha$ inhibitors the expression level of miRNA-5196 was also reduced and reached almost HC sera level (1.27-fold, $p=0.117)$. This suggests that expression of circulating miRNA-5196 from patients suffering from rheumatic diseases including SSc, RA and AS is significantly elevated and it can be reduced by anti-TNF- $\alpha$ treatment.

\section{Treatment with Biologic Therapy is Accompanied with Significant Down-Regulation of MiRNA-5196 Expression but not with Reduction of CRP Level}

Apart from two individuals, the expression of miRNA5196 was significantly decreased (2.16-fold, $p=0.024)$ in 


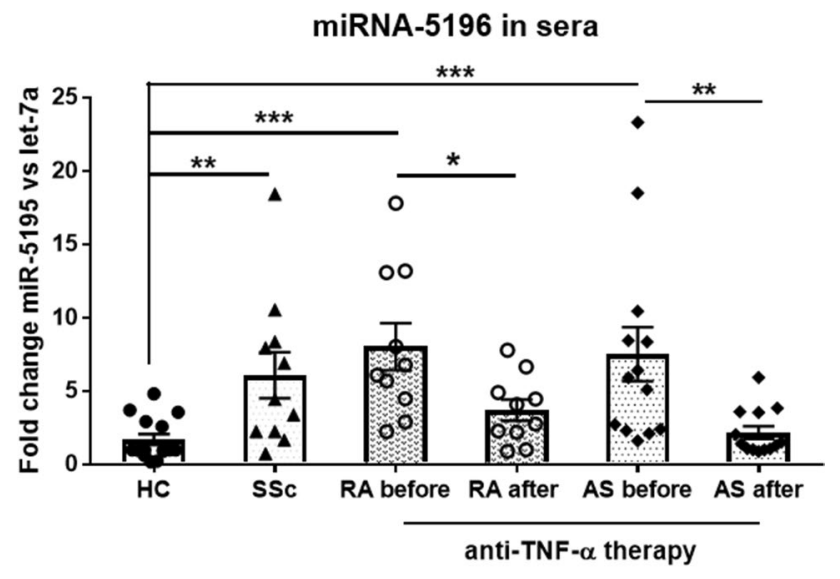

Fig. 1 Level of circulating miRNA-5196 present in sera of rheumatic disease patients. The expression level of sera circulating miRNA-5196 was measured in HC $(n=15)$, SSc $(n=11)$ patients and in RA patients before $(n=10)$ and after $(n=10)$ TNF-a therapy and in AS patients before $(n=13)$ and after $(n=13)$ TNF-a therapy. Results were normalized to the let-7a internal control. Each symbol represents an individual subject; horizontal lines with bars show the mean \pm SEM. $P$ values are expressed as follows: $0.05>P>0.01$ as *; $0.01>P>0.001$ as $* * ; P<0.001$ as $* * *$

all RA patients following anti-TNF- $\alpha$ treatment (Fig. 2a). Simultaneously, the average DAS28 score was also significantly reduced $(p<0.0001)$ from 6.77 to 4.48 in RA patients who were undergoing 6 months biologic therapy (Fig. 2b) whereas inflammation parameter including CRP did not drop significantly ( $p=0.67$ ) (Fig. 2c). Regarding AS patients we have also noticed significant reduced expression of miRNA5196 (3.45-fold, $p=0.009$ ) (Fig. 3a) and ASDAS (1.59-fold, $p=0.004$ ) (Fig. 3b) following biologic agent treatment. Similarly to RA patients, the CRP level in AS patients did not drop significantly ( $p=0.20$ ) (Fig. 3c). Thus, we speculate that reduction in miRNA-5196 expression, but not decreased level of CRP, might a good marker of anti-TNF- $\alpha$ therapy response in RA and AS patients.

\section{Changes in MiRNA-5196 Expression Can be Used as a Good Marker for Predicting Reduced Disease Activity Score upon TNF-a Inhibitors Treatment in RA and AS Patients}

In addition, we have also calculated delta miRNA-5196 expression and delta CRP based on values just before and after anti-TNF- $\alpha$ treatment. Subsequently, these results were correlated with delta DAS28 to compare which parameter, either delta miRNA-5196 or delta CRP, will yield better outcome of anti-TNF- $\alpha$ therapy in RA and AS patients. Interestingly, we noticed that delta miRNA-5195 expression positively correlates with delta DAS28 $(p=0.039, r=0.67)$ (Fig. 4a), whereas changes in CRP level did not correlate with delta DAS28 in RA patients $(p=0.38, r=-0.30)$ (Fig. 4b). Of note, we observed that delta CRP revealed even a negative trend with delta DAS28 $(p=0.38, r=-0.30)$ (Fig. 4b) and delta miRNA-5196 ( $p=0.42, r=-0.28)$ (Fig. 4c). In AS patients, delta miRNA-5196 expression was also positively correlated $(p=0.02, r=0.75)$ with delta ASDAS (Fig. 5a) and delta CRP ( $p=0.04, r=0.69)$ (Fig. 5c). As previously shown for RA, delta CRP did not correlate with delta ASDAS ( $p=0.16, r=0.50$ ) (Fig. 5b). These results strongly suggest that changes in miRNA-5196 expression serve as a better predictive biomarker of antiTNF- $\alpha$ response than changes in CRP level both in RA and AS patients. Due to a small change in miRNA-5196 expression before and after biologic therapy, we have excluded four patients' samples from the analysis. In Fig. 5, we eliminated the delta miRNA-5196 samples which were within the

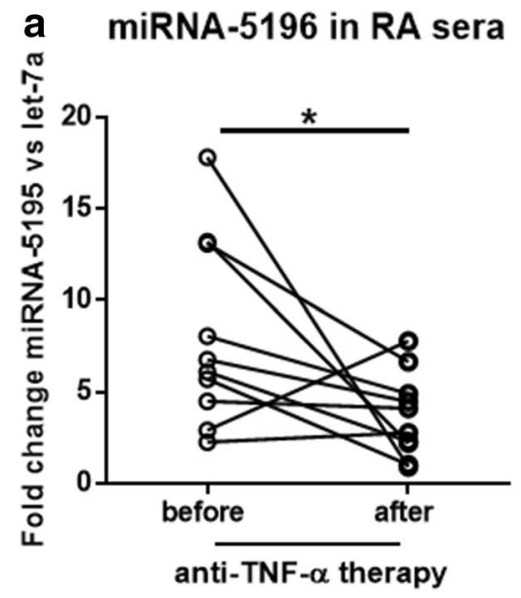

Fig. 2 Expression level of miRNA-5196, DAS28 score and CRP before and after anti-TNF-a therapy. The expression level of sera circulating miRNA-5196 (a), disease activity score DAS28 (b) and CRP
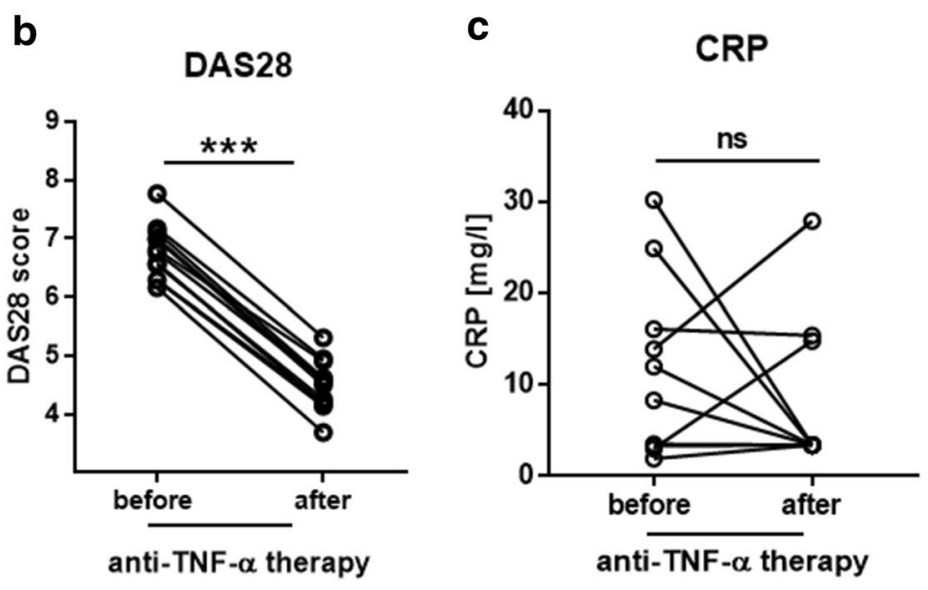

(c) were measured in RA patients before $(n=10)$ and after $(n=10)$ TNF-a therapy. $P$ values are expressed as follows: $005>P>001$ as *; $001>\mathrm{P}>0001$ as $* * ; P<0001$ as $* * *$, ns not significant 

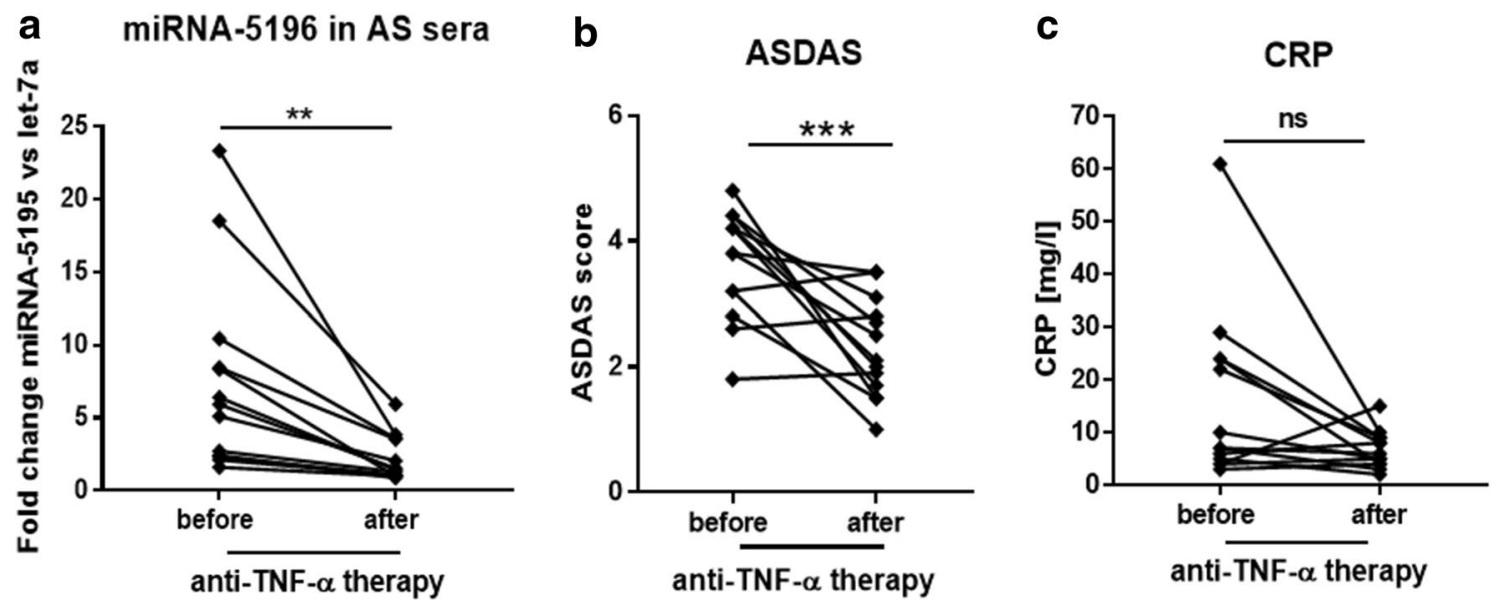

Fig. 3 Expression level of miRNA-5196, DAS28 score and CRP before and after anti-TNF-a therapy. The expression level of sera circulating miRNA-5196 (a), ankylosing spondylitis disease activity score ASDAS (b) and CRP (c) were measured in AS patients before $(n=13)$ and after $(n=13)$ TNF-a therapy. $P$ values are expressed as follows: $005>P>001$ as *; $001>P>0001$ as **; $P<0001$ as $* * *$, us not significant

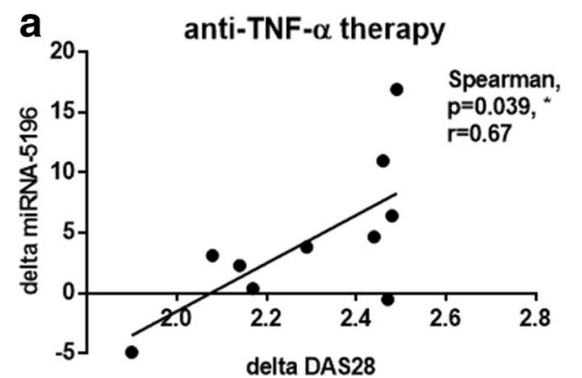

b

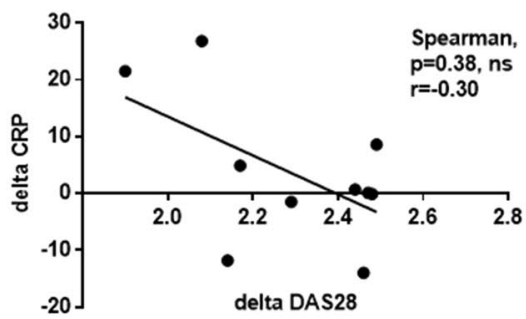

C

anti-TNF- $\alpha$ therapy

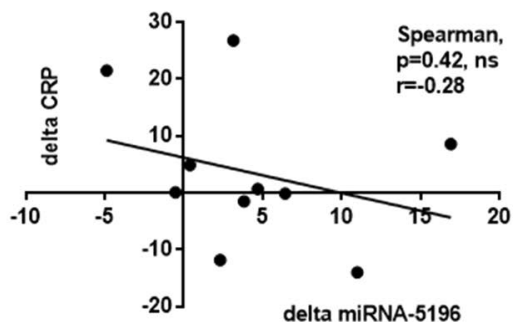

Fig. 4 Correlation between changes in serum level of miRNA-5196 (delta miRNA-5196), changes in DAS28 (delta DAS28) and changes in CRP levels (delta CRP) in RA patients $(n=10)$ following antiTNF-a therapy. Changes in miRNA-5196 expression were correlated with changes in DAS28 (a), changes in CRP levels were correlated with changes in DAS28 (b) and changes in CRP levels were correlated with changes in miRNA-5196 expression (c)

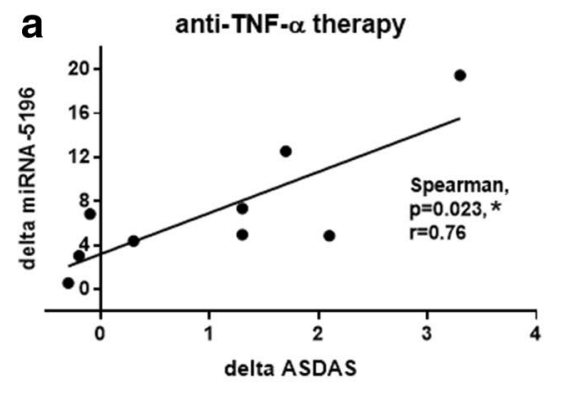

b

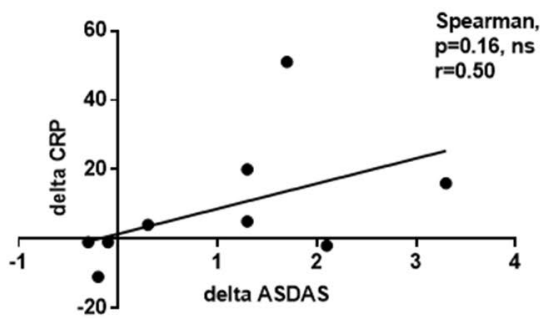

C

anti-TNF- $\alpha$ therapy

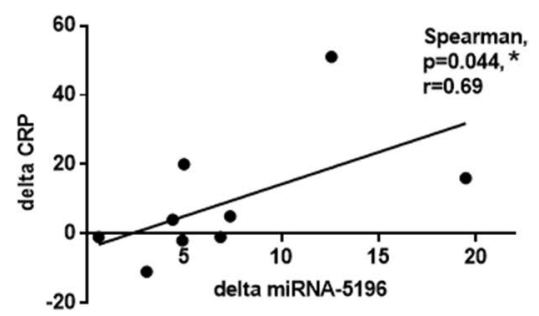

Fig. 5 Correlation between changes in serum level of miRNA5196 (delta miRNA-5196), changes in ASDAS (delta ASDAS) and changes in CRP levels in AS patients $(n=9)$ following anti-TNF-a therapy. Changes in miRNA-5196 expression were correlated with changes in ASDAS (a), changes in CRP levels were correlated with changes in ASDAS (b) and changes in CRP levels were correlated with changes in miRNA-5196 expression (c) 
range between 1 and 1.5. In RA patients, delta miRNA-5196 was substantial and always out of this range; therefore, all patients were included in the correlation analysis (Fig. 4).

\section{Delta MiRNA-5196 is a More Accurate Biomarker to Monitor Changes of Clinical Activity of AS and RA Patients than Delta CRP}

Finally, to evaluate the diagnostic potential of circulating miRNA-5196 as a useful detection biomarker predicting response to a biologic therapy, ROC curve analysis was performed. In Fig. 6a, b, it can be seen that delta miRNA-5196 was much more specific in predicting anti-TNF- $\alpha$ response (area under curve $(\mathrm{AUC})=0.87, \mathrm{CI}=0.63-1.11, p=0.055$ and $\mathrm{AUC}=0.90, \mathrm{CI}=0.67-1.21, p=0.050$, respectively, in RA and AS patients) than delta CRP level (AUC $=0.83$, $\mathrm{CI}=0.56-1.10, p=0.088$ and $\mathrm{AUC}=0.85, \mathrm{CI}=0.56-1.13$, $p=0.086$, respectively, in RA and AS patients). These results indicate that sera miRNA-5196 can be a better biomarker to measure reduced disease activity upon biologic therapy treatment than CRP level both in RA and AS patients. Based on the Youden Index, at a cut-off value of 3.81 for delta miRNA-5196, sensitivity and specificity values were 83 and $100 \%$, respectively, for RA patients, whereas at a cutoff value of 0.7 for delta CRP, sensitivity and specificity values were 83 and 75\%, respectively. In AS patients, at a cut-off value of 4.89 for delta miRNA-5196, sensitivity and specificity values were 100 and $75 \%$, respectively, for RA, whereas at a cut-off value of 5 for delta CRP, sensitivity and specificity values were 80 and $100 \%$, respectively.

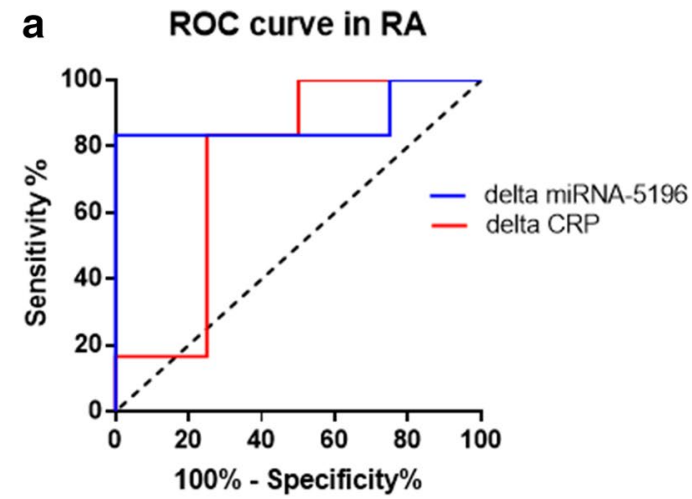

\begin{tabular}{|c|c|c|c|c|}
\hline Biomarker & AUC & SE & p value & 95\% Cl \\
\hline $\begin{array}{c}\text { miRNA- } \\
5196\end{array}$ & 0.87 & 0.124 & 0.055 & $\begin{array}{c}0.63 \text { to } \\
1.11\end{array}$ \\
\hline CRP & 0.75 & 0.178 & 0.201 & $\begin{array}{c}0.38 \text { to } \\
1.11\end{array}$ \\
\hline
\end{tabular}

\section{Discussion}

In this study, we investigated whether sera circulating miRNA-5196 can be used as a versatile biomarker characterising autoimmune rheumatic diseases including RA, SSc and AS. In addition, we examined whether changes in miRNA-5196 expression can be used as a better predictor for clinical response to anti-TNF- $\alpha$ therapy than changes in CRP levels in RA and AS patients.

RA is a complex autoimmune disease characterized by inflammation and joint destruction. Similarly, AS is characterized by joint inflammation that primarily affects the spine. Overall, both diseases result in stiffness, pain and joint deformities. Although, the management of rheumatic diseases has undergone major advances in recent years, both in terms of the drugs discovery and therapeutic strategy but still pose a significant socioeconomic problem (Klak et al. 2016). Unfortunately, current treatment still does not guarantee complete recovery of patients with rheumatic diseases and it is only possible to slow down or reduce the disease activity. Thus, the development of more effective diagnosis of rheumatic diseases are badly needed to increase patients' functioning and quality of life. Inflammation in RA and AS is mediated mostly by TNF- $\alpha$; therefore, the introduction of biologic therapy targeting this cytokine has revolutionised RA and AS treatment. Unfortunately, high costs of anti-TNF- $\alpha$ treatment requires good predictors to reduce the economic burden of RA treatment. Indeed, there are currently no straightforward

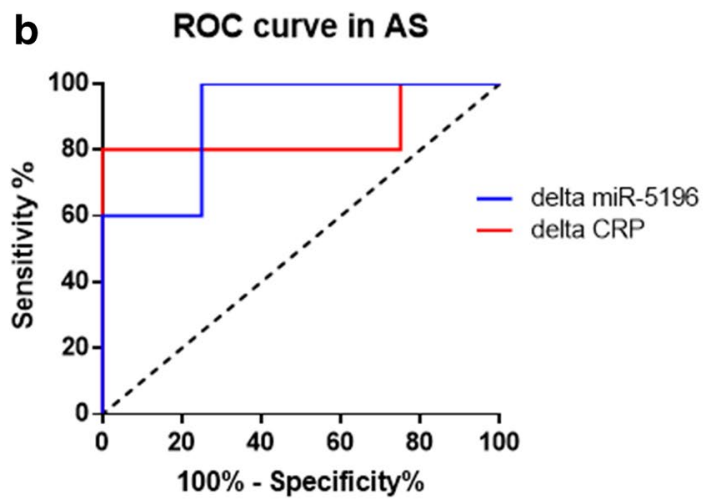

\begin{tabular}{|c|c|c|c|c|}
\hline Biomarker & AUC & SE & p value & $\mathbf{9 5 \%} \mathbf{~ C l}$ \\
\hline $\begin{array}{c}\text { miRNA- } \\
5196\end{array}$ & 0.90 & 0.112 & 0.050 & $\begin{array}{c}0.67 \text { to } \\
1.12\end{array}$ \\
\hline CRP & 0.85 & 0.146 & 0.086 & $\begin{array}{c}0.56 \text { to } \\
1.13\end{array}$ \\
\hline
\end{tabular}

Fig. 6 ROC analysis comparing delta miRNA-5196 and delta CRP levels as biomarkers predicting anti-TNF-a outcomes in RA (a) and AS patients (b). 10 RA patients and 9 AS patients were included in the analysis 
predictors of a good response to anti-TNF- $\alpha$ drugs and significant percentage of patients fail to respond to treatment (25-38\% of etanercept patients, $21-42 \%$ of infliximab patients) (Seymour et al. 2001). Recent studies have shown that miRNA play an important role in RA and AS diagnosis and dysregulated miRNA expression seems to contribute to the molecular mechanisms of the disease (Chen et al. 2015; Li et al. 2016; Prajzlerova et al. 2017). MiRNAs are short (approximately 21 nucleotides long) non-coding RNAs that can influence mRNA processing at the post-transcriptional level. It has been shown that plasma miRNA signature of miRNA-23 and miRNA-223 was used as predictor and biomarker of response to antiTNF- $\alpha$ /DMARDs combination therapy in RA patients (Castro-Villegas et al. 2015). Indeed, upregulated expression of miRNA-23 and miRNA-223 was inversely correlated with secretion of TNF- $\alpha$, IL-6, IL-17, rheumatoid factor and CRP in responder patients (Castro-Villegas et al. 2015). Expression of miRNA-451 in T cells also positively correlated with DAS28 (Smigielska-Czepiel et al. 2014). Another study has shown that expression of miRNA-125b in peripheral blood mononuclear cells was a better predictor for an optimal treatment outcome after 3 months TNF- $\alpha$ therapy than baseline levels of DAS28, CRP, erythrocyte sedimentation rate (ESR) (Hruskova et al. 2016). In the recent studies, it has been observed that sera circulating miRNA-29a-3p, miRNA-146a-5p or miRNA-222-3p were associated with spinal changes and/or disease activity assessed by bath ankylosing spondylitis disease activity index (BASDAI) in AS patients (Prajzlerova et al. 2017). Interestingly, AS patients receiving anti-TNF- $\alpha$ therapy exhibited significantly lower levels of selected miRNAs than anti-TNF- $\alpha$ naive patients (Prajzlerova et al. 2017). Previously, we have also demonstrated that miRNA-135b targeting STAT3 is reduced in other rheumatic diseases including SSc (O'Reilly et al. 2016). Whereas, miRNA-5196 is elevated in SSc monocytes and SSc sera, suggesting that these miRNAs can be used as potential biomarkers characterising SSc. In addition, high-throughput Solexa deep sequencing analysis followed by computational analysis identifies miRNA-5196 as one of novel candidates characterising different types of childhood acute lymphoblastic leukemia (ALL) (Schotte et al. 2011). Indeed, miRNA-5196 has been listed as a top 10 of novel miRNAs with highest read frequency in ALL patients. Similarly, miRNA-5196 was upregulated in esophageal cancer (Liao et al. 2016). In contrast, the expression of miRNA-5196 in serum of colon cancer patients was downregulated compared to HC suggesting that circulating miRNA-5196 may be applied in early tumor diagnosis, prognosis and recurrence with a great value (Liu et al. 2015; Zhang et al. 2017). Based on prediction algorithms obtained from miRDB, Target Scan
Human and miRanda databases, miRNA-5196 has been described as a negative regulator of Fra2 (AP-1 family transcription factor) and MMP-15, IL-1 receptor type I. These molecules play an important role in inflammation and progression of rheumatic diseases (Araki and Mimura 2017; Dey et al. 2016; Magyari et al. 2014). Indeed, in the previous paper we have shown that miRNA-5196 targets Fra2 and subsequently reduces profibrotic TIMP-1 production in SSc monocytes (Ciechomska et al. 2017). MiRNA5196 binds to five seed regions within 3'UTR of Fra2, therefore, can negatively regulate gene expression of target mRNA (Ciechomska et al. 2017). Thus, we sought to investigate whether miRNA-5196 could be used as a novel biomarker for predicting anti-TNF- $\alpha$ therapy in RA and AS patients. Indeed, in the present study we have shown that sera circulating miRNA-5196 is elevated in SSc, RA and AS patients compared to HC (Fig. 1). Following antiTNF- $\alpha$ therapy, the level of miRNA-5196 was reduced in RA patients and in AS patients. Reduced expression of miRNA-5196 was seen in $80 \%$ of RA patients responding to anti-TNF- $\alpha$ therapy and in all AS patients (Fig. 2). Similar to the previously published results (Ciechomska et al. 2017), we have also seen increased expression of miRNA5196 in SSc sera (Fig. 1). In addition, we have demonstrated that a change in miRNA-5196 expression positively correlates with changes in clinical parameters (DAS28 and ASDAS) upon biologic therapy treatment in RA and AS patients, respectively (Figs. 4a, 5a). In contrast, in Figs. 4b and $5 \mathrm{~b}$, it can be seen that alteration in the CRP levels did not associate with clinical parameters in RA and AS patients. These results suggests that changes in miRNA5196 expression occurred during TNF- $\alpha$ therapy could support more informed clinical decisions on the most appropriate treatment regimens for individual RA and AS patients than changes in CRP levels. Although changes in the CRP levels are considered as a gold standard to evaluate therapy in rheumatic diseases, some studies have shown controversial role of CRP level as a biomarker for disease activity. In systemic lupus erythematosus patients there were no correlations between CRP serum levels or anti-CRP antibodies and disease activity (Rezaieyazdi et al. 2011; Son et al. 2017), suggesting that CRP may not be an ideal indicator for disease activity. Furthermore, using ROC analysis evaluating the diagnostic accuracy of biomarkers, we have observed that delta miRNA-5196 was a better predictor for a clinical response to personalised biologic therapy than delta CRP in RA and AS patients. Indeed, we have observed larger AUC values for delta miRNA-5196 (0.87 for RA and 0.90 for AS) compared to AUC values for delta CRP ( 0.75 for RA and 0.85 for AS).

Taken together, we have shown that changes in miRNA5196 expression holds promise as a good marker of antiTNF- $\alpha$ treatment response. A clear limitation of our study is 
a relatively small sample size of the groups. Ideally, validation of miRNA-5196 expression as a prognostic marker in large cohorts of patients may allow for better selection of RA and AS patients benefiting the most from anti-TNF- $\alpha$ therapy. Therefore, more studies exploring the value of miRNA5196 as a predictive biomarker for treatment response would be of interest and may facilitate their introduction into clinical practice.

Acknowledgements This work was supported by Homing Plus grant/2013-8/4 from Foundation for Polish Science and EU structural funds (MC), 2015/16/S/NZ6/00041 (MC) and 2016/21/B/NZ5/01901 (KBK) grants from National Science Centre, Poland. The authors thank the Regional Centre of Transfusion Medicine and Blood Bank in Wroclaw and Warsaw for providing control samples. We would like to thank Malgorzata Manczak for helping with ROC analysis.

\section{Compliance with Ethical Standards}

Conflict of Interest The authors declare that they have no conflicts of interest.

Open Access This article is distributed under the terms of the Creative Commons Attribution 4.0 International License (http://creativeco mmons.org/licenses/by/4.0/), which permits unrestricted use, distribution, and reproduction in any medium, provided you give appropriate credit to the original author(s) and the source, provide a link to the Creative Commons license, and indicate if changes were made.

\section{References}

Aletaha D, Neogi T, Silman AJ et al (2010) 2010 Rheumatoid arthritis classification criteria: an American College of Rheumatology/ European League Against Rheumatism collaborative initiative. Arthritis Rheum 62:2569-2581

Alevizos I, Illei GG (2010) MicroRNAs as biomarkers in rheumatic diseases. Nat Rev Rheumatol 6:391-398

Araki Y, Mimura T (2017) Matrix metalloproteinase gene activation resulting from disordred epigenetic mechanisms in rheumatoid arthritis. Int J Mol Sci 18. pii: E905

Bao C, Huang F, Khan MA et al (2014) Safety and efficacy of golimumab in Chinese patients with active ankylosing spondylitis: 1-year results of a multicentre, randomized, double-blind, placebo-controlled phase III trial. Rheumatology 53:1654-1663

Brennan FM, Chantry D, Jackson A et al (1989) Inhibitory effect of TNF alpha antibodies on synovial cell interleukin-1 production in rheumatoid arthritis. Lancet 2:244-247

Castro-Villegas C et al (2015) Circulating miRNAs as potential biomarkers of therapy effectiveness in rheumatoid arthritis patients treated with anti-TNFalpha. Arthritis Res Ther 17:49

Catrina AI, Lampa J, Ernestam S et al (2002) Anti-tumour necrosis factor (TNF)-alpha therapy (etanercept) down-regulates serum matrix metalloproteinase (MMP)-3 and MMP-1 in rheumatoid arthritis. Rheumatology 41:484-489

Chen XM, Huang QC, Yang SL et al (2015) Role of micro RNAs in the pathogenesis of rheumatoid arthritis: novel perspectives based on review of the literature. Medicine 94:e1326
Ciechomska M, O'Reilly S (2016) Epigenetic modulation as a therapeutic prospect for treatment of autoimmune rheumatic diseases. Mediators Inflamm 2016:9607946

Ciechomska M, O'Reilly S, Suwara M et al (2014) MiR-29a reduces TIMP-1 production by dermal fibroblasts via targeting TGF-beta activated kinase 1 binding protein 1, implications for systemic sclerosis. PLoS One 9:e115596

Ciechomska M, Zarecki P, Merdas M et al (2017) The role of microRNA-5196 in the pathogenesis of systemic sclerosis. Eur J Clin Invest 47:555-564

Curtis JR, Singh JA (2011) Use of biologics in rheumatoid arthritis: current and emerging paradigms of care. Clin Ther 33:679-707

Davoren PA, McNeill RE, Lowery AJ et al (2008) Identification of suitable endogenous control genes for microRNA gene expression analysis in human breast cancer. BMC Mol Biol 9:76

Dey P, Panga V, Raghunathan S (2016) A cytokine signalling network for the regulation of inducible nitric oxide synthase expression in rheumatoid arthritis. PLoS One 11:e0161306

Dolcino M, Tinazzi E, Pelosi A et al (2017) Gene expression analysis before and after treatment with adalimumab in patients with ankylosing spondylitis identifies molecular pathways associated with response to therapy. Genes 8(4):127

Gibofsky A (2012) Overview of epidemiology, pathophysiology, and diagnosis of rheumatoid arthritis. Am J Manag Care 18(13 Suppl):S295-S302

Hruskova V, Jandova R, Vernerova L et al (2016) MicroRNA-125b: association with disease activity and the treatment response of patients with early rheumatoid arthritis. Arthritis Res Ther 18:124

Inman RD, Davis JC Jr, Dv H et al (2008) Efficacy and safety of golimumab in patients with ankylosing spondylitis: results of a randomized, double-blind, placebo-controlled, phase III trial. Arthritis Rheum 58:3402-3412

Klak A, Raciborski F, Samel-Kowalik P (2016) Social implications of rheumatic diseases. Reumatologia 54:73-78

Li Y, Zhang L, Liu F et al (2015) Identification of endogenous controls for analyzing serum exosomal miRNA in patients with hepatitis B or hepatocellular carcinoma. Dis Markers 2015:893594

Li Z, Wong SH, Shen J et al (2016) The role of MicroRNAS in ankylosing spondylitis. Medicine 95:e3325

Liao J, Liu R, Shi YJ et al (2016) Exosome-shuttling microRNA-21 promotes cell migration and invasion-targeting PDCD4 in esophageal cancer. Int J Oncol 48:2567-2579

Liu J, Yan J, Zhou C et al (2015) miR-1285-3p acts as a potential tumor suppressor miRNA via downregulating JUN expression in hepatocellular carcinoma. Tumour Biol 36:219-225

Macfarlane LA, Murphy PR (2010) MicroRNA: biogenesis, function and role in cancer. Curr Genomics 11:537-561

Magyari L, Varszegi D, Kovesdi E et al (2014) Interleukins and interleukin receptors in rheumatoid arthritis: research, diagnostics and clinical implications. World J Orthop 5:516-536

Maini RN, Feldmann M (2002) How does infliximab work in rheumatoid arthritis? Arthritis Res 4(Suppl 2):S22-S28

Mendell JT, Olson EN (2012) MicroRNAs in stress signaling and human disease. Cell 148:1172-1187

Nair SC, Welsing PM, Choi IY et al (2016) A personalized approach to biological therapy using prediction of clinical response based on MRP8/14 serum complex levels in rheumatoid arthritis patients. PLoS One 11:e0152362

O'Reilly S, Ciechomska M, Fullard N et al (2016) IL-13 mediates collagen deposition via STAT6 and microRNA-135b: a role for epigenetics. Sci Rep 6:25066

Prajzlerova K, Grobelná K, Hušáková M et al (2017) Association between circulating miRNAs and spinal involvement in patients with axial spondyloarthritis. PLoS One 12:e0185323 
Rezaieyazdi Z, Sahebari M, Hatef MR et al (2011) Is there any correlation between high sensitive CRP and disease activity in systemic lupus erythematosus? Lupus 20:1494-1500

Rudwaleit M, van der Heijde D, Landewé R et al (2009) The development of Assessment of SpondyloArthritis international Society classification criteria for axial spondyloarthritis (part II): validation and final selection. Ann Rheum Dis 68:777-783

Schoels M, Wong J, Scott DL et al (2010) Economic aspects of treatment options in rheumatoid arthritis: a systematic literature review informing the EULAR recommendations for the management of rheumatoid arthritis. Ann Rheum Dis 69:995-1003

Schotte D, Akbari Moqadam F, Lange-Turenhout EA et al (2011) Discovery of new microRNAs by small RNAome deep sequencing in childhood acute lymphoblastic leukemia. Leukemia 25:1389-1399

Seymour HE, Worsley A, Smith JM et al (2001) Anti-TNF agents for rheumatoid arthritis. Br J Clin Pharmacol 51:201-208

Smigielska-Czepiel K, van den Berg A, Jellema P et al (2014) Comprehensive analysis of miRNA expression in T-cell subsets of rheumatoid arthritis patients reveals defined signatures of naive and memory Tregs. Genes Immun 15:115-125

Son CN, Lee TH, Bang JH et al (2017) The relationship between anti-C-reactive protein and disease activity in patients with systemic lupus erythematosus. Korean J Intern Med. https://doi. org/10.3904/kjim.2016.065

Taylor PC, Feldmann M (2009) Anti-TNF biologic agents: still the therapy of choice for rheumatoid arthritis. Nat Rev Rheumatol 5:578-582

Wang Y, Wang H, Jiang J et al (2016) Comparative efficacy and acceptability of anti-TNF-alpha therapy in ankylosing spondylitis: a mixed-treatments comparison. Cell Physiol Biochem 39:1679-1694

Zhang Y, Li M, Ding Y et al (2017) Serum microRNA profile in patients with colon adenomas or cancer. BMC Med Genom 10:23 